\title{
Biomechanically Optimized Distributed Tactile Transducer Based on Lateral Skin Deformation
}

\author{
Qi Wang and Vincent Hayward
}

Haptics Laboratory, Center for Intelligent Machines, McGill University, Montréal, Canada*

\begin{abstract}
This paper presents a tactile transducer device that is optimized from biomechanical data and has a compact, yet modular design. The tactile transducer comprises a $6 \times 10$ piezo bimorph actuator array with a spatial resolution of $1.8 \times 1.2$ millimeters and a wide temporal bandwidth. The actuator mounting method was improved from a conventional cantilever method to a dual-pinned method, giving the actuator the capability of maximally deforming glabrous skin for laterotactile stimulation. The results were validated using a psychophysical method. The tactile display device is modular, appeals to ordinary fabrication methods, and can be assembled and dismantled in a short time for debugging and maintenance. It weighs $60 \mathrm{~g}$, it is self-contained in a $150 \mathrm{~cm}^{3}$ volume and may be interfaced to most computers, provided that two analog outputs and six digital IO lines are available. Psychophysical experiments were carried out to assess its effectiveness in rendering virtual tactile features.
\end{abstract}

\section{Introduction}

Much of our extraordinary manipulation skills rely on the sense of touch, which is particularly developed in the fingertips. In our daily lives, we depend very much on tactile sensations to guide manipulation and to perceive the outside world; for instance, when doing up laces, discriminating unseen objects in a pocket, or localizing lumps in the body. In some instances, such as in minimally invasive surgery, surgeons must work with di-

\footnotetext{
${ }^{*}$ Q. Wang is now with the Wallace H. Coulter Department of Biomedical Engineering at Georgia Tech and Emory University V. Hayward is now with UPMC Univ Paris 06, UMR 7222, Institut des Systèmes Intelligents et de Robotique, F-75005, Paris, France. A portion of this paper appeared in the proceedings of the Symposium on Haptic Interfaces for Virtual Environments and Teleoperator Systems, 2006.
}

minished or even absent tactile sensations. The ability to artificially deliver tactile signals as conveniently and economically as graphic or sonic signals is much needed. Artificial tactile feedback would be of valuable assistance in such situations as tele-operation, virtual environment simulations, or medical training.

Tactile displays may be viewed as computer peripheral devices meant to create computer-generated tactile sensations similar to those arising when holding objects, palpating them, or sliding fingers on their surfaces. The design and fabrication of these tactile transducers has received much attention from researchers, despite the difficulties encountered in achieving systems that are robust, effective, manufacturable, and of reasonable complexity.

This problem has been approached in various ways. Attempts have been made to leverage the benefits of smart actuators such as shape memory alloy wires or springs (Wellman et al., 1998), (Velazquez et al., 2005), miniature electrostatic actuators (Jungmann and Schlaak, 2002), electroactive gels (Konyo et al., 2000), specifically developed technologies such as pneumatic bladders (Moy et al., 2000) and miniature electromagnetic solenoids (BenaliKhoudja et al., 2004), even hobby servo-motors (Wagner et al, 2004), or remotized miniature DC motors (Sarakoglou et al., 2005).

Another direction of investigation is characterized by experimentation with new techniques for interaction with the skin that include tapping on it (Bliss et al., 1970), brushing against it (Ghodssi et al., 1998), electrostatic adhesion (Tang and Beebe, 1998), or ultrasonic pressure (Iwamoto and Shinoda, 2005). For a survey, please see (Pasquero, 2006).

In the past few years, our laboratory has been developing several tactile displays that deform the skin by tangential traction (Hayward and Cruz-Hernandez, 2000; Pasquero and Hayward, 2003), a principle we termed laterotactile stimulation (Pasquero et al., 2007). The 
idea is to design a display system that produces skin deformations that resemble those experienced by the fingerpad skin during contact with objects, rather than attempting to re-create the cause of these deformations. Recently, there has been increasing evidence of the effectiveness of this approach to create the experience of small shapes (Levesque et al., 2005) or weak surface undulation (Kikuuwe et al., 2005). At the same time, skin lateral deformation is being studied for its perceptual contributions to the design of displays (Biggs and Srinivasan, 2002; Drewing et al., 2005).

Three successive designs, which are characterized by increasing performance and diminishing complexity, have been developed to date, as shown in Fig. 1.

In the initial design represented in Figs. 1(a,b), a dense array of piezoelectric actuators were arranged vertically, and operated in $d_{31}$ mode (electric field applied laterally, mechanical deformation along the vertical direction) (Hayward and Cruz-Hernandez, 2000). The actuators were bonded to a deformable sub-micrometric membrane with specific elastic characteristics to allow deformation to occur in two directions. When the membrane deforms, the vertical tubes bonded to the upper surface of the membrane swing laterally, also in two directions. The main difficulties encountered with this device were the fabrication of the membrane and the final assembly. While these difficulties were overcome, the intensity of the tactile images were too low to be generally useful. This weakness was attributed to the compliance of the structure and the limited free deflection of the contactors.

These initial results led us to consider a "stacked comb" design for the STRESS device illustrated in Figs. 1(c,d) (Pasquero and Hayward, 2003). Actuators were fabricated by ten solid bimorph piezoelectric plates (Y poled), which greatly simplified assembly. As the bimorph plates are comprised of two piezo ceramic sheets bonded together, the bending stimulation is achieved by expanding one piezo sheet and contracting the other sheet. While this design allowed us to verify that, indeed, when deformation patterns are created on the skin with millimetric scale resolution, the exact characteristics of the local strain did not seem to make a difference on the resulting conscious experience, only large scale patterns mattered. Despite significant improvement in the intensity of the stimuli, these were still found too weak for the average user. Also the device assembly was hard due to the numerous electrical connections that had to be made in small space. But perhaps the greatest problem was robustness. Should one or several ac-

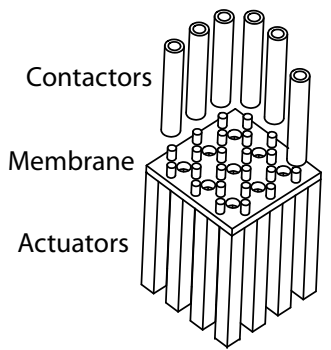

(a)

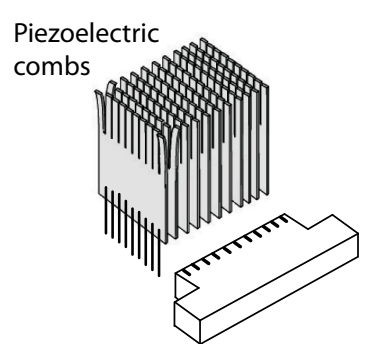

(c)

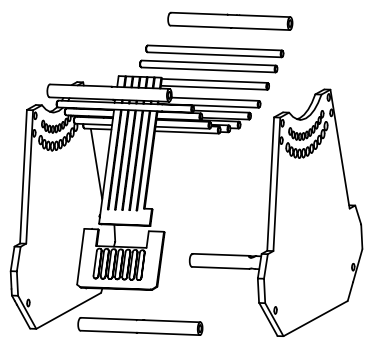

(e)

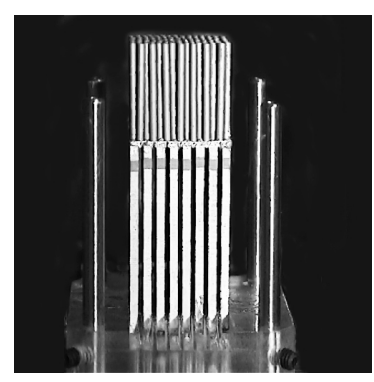

(b)

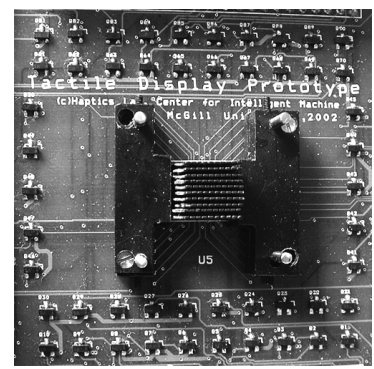

(d)

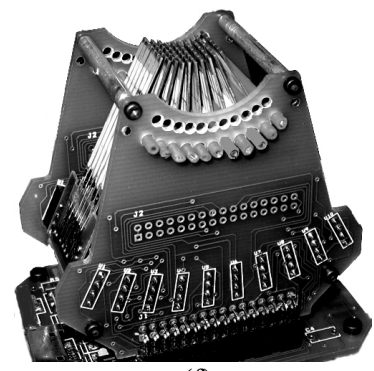

(f)
Figure 1: (a,b) Membrane architecture described in (Hayward \& Cruz, 2000). (c,d) STRESS architecture as in (Pasquero \& Hayward, 2003). (e,f) Modular design as in this paper. To give scale, in all cases the active surface is about $1 \mathrm{~cm}^{2}$.

tuators be damaged or fail, the entire device had to be reconstructed from scratch.

This paper describes a third generation, high performance tactile display device named STRESS ${ }^{2}$. These developments were motivated by an assiduous pursuit of complexity reduction, both in the fabrication and the assembly methods, while still boosting performance. Good performance was the result of two key improvements. First, a modular architecture shown in "exploded view" in the Fig. 1(e,f) was introduced. Each actuator module could be manufactured separately and plugged into two 
lateral printed circuit boards which provide both electrical connections and structural strength. Second, we introduced a new piezo actuator mounting technique, termed dual-pinned lever, which was shown to be capable of maximally stimulating the fingerpad skin given an actuator operating in bending mode, keeping in mind that, in tactile display design, actuation is invariably a limiting factor.

\section{Optimization}

\subsection{Approach}

To find a preferred configuration and geometry, we sought to maximize the strain created by the display where the mechanorecpetors are located inside the skin, since it would maximize stimulation. The display operates by traction at the skin surface. Therefore, the question was to establish a relationship between surface strain and strain deeper inside the skin. Such study was performed by the authors under the assumption that the skin behaved like a homogenous material (Wang and Hayward, 2008). In the later study, it was found that the virtual height, $A$, of a displayed feature is a function of the lineic force that creates traction, $Q$, according to $A=0.37 Q / E$, where $E$ is the young modulus of the linear material. Notice that this expression does not involve information regarding the location of the targeted mechanoreceptors. On the other hand, the preferred spatial period, $g_{\text {opt }}$, of the traction surfaces was found to be directly related to the depth at which the receptors are located, according to a law that is, by and large, linear in the range considered. The preferred spacing is independent from the Young modulus of the considered material: $g_{\mathrm{opt}}=2 d_{\mathrm{opt}} \approx 1.2 Z$, where $Z$ is the depth of targeted receptors, highlighting the purely geometrical contraints involved in the design of tactile displays. Based on considerations regarding the skin anatomy discussed in (Wang and Hayward, 2008), we took $g=1.2 \mathrm{~mm}$.

Of course these findings depend on the assumptions of homogeneity and linearity of the mechanics of the skin. That the skin is actually nonlinear is a critical question for display design, particularly because of nonlinear stiffening. In order to address this problem, we made the assumption that the skin could be approximated by a nonlinear homogenous material with a known range of 'effective' or 'equivalent' Young moduli, measured in the condition of lateral surface traction. We then sought to find the best geometry for a bending actuator. In an- other experiment, we sought to find the geometry that caused maximum stimulation using a psychophysical approach. To this end, we asked a group of volunteers to employ the method of adjustments in order to find the geometry that maximized the intensity of the sensation for a given input voltage. These distinctively different methods gave similar results, vindicating our initial assumption that strain inside the skin is the determinant perceptual factor for standard signals, despite the numerous approximations that were made.

\subsection{Constituent equations of dual- pinned piezoelectric bimorph ben- ders}

We first consider the constituent equation of a cantilever-mounted parallel bimorph bender, Fig. 2(a), (Smits et al. 1991),

$$
\delta=\frac{l^{3}}{2 E w h^{3}} f^{z}+\frac{3 d_{31} l^{2}}{4 h} E_{3},
$$

where $\delta$ is the tip deflection, $E$, is the material Young's modulus, $l, w$, and $h$, the length, width, and thickness of the bender's layers respectively, $f^{z}$ the force applied at the tip, $d_{31}$ the piezoelectric coefficient, and $E_{3}$ is the applied electric field. The compliance and the free deflection (the deflection with no external load) are,

$$
\frac{\partial \delta}{\partial f^{z}}=\frac{l^{3}}{2 E w h^{3}} \quad \text { and } \quad \delta_{\text {free }}=\frac{3 d_{31} l^{2}}{4 h^{2}} V_{\max }
$$

These expressions show that the free deflection varies with $l^{2}$ and the compliance with $l^{3}$. For a given compliant load, there must exist an optimal length $l_{\text {opt }}$ that maximizes deflection. With the best commercially available piezoelectric materials, we found that a cantilever could not deform the skin in the desired large range (i.e. 30-50\%), even with a carefully selected $l_{\text {opt }}$. For a sufficiently large free deflection the actuator would be too soft and for the necessary stiffness, it would not deflect enough.

If instead benders are clamped at two places as indicated in Fig. 2(b), this new structure, termed "dualpinned lever" will always produce a higher blocked force for an equivalent deflection. By varying the ratio $l_{1} / l_{2}$ it is also possible to trade more stiffness for less free deflection. In this study, we tuned our device for glabrous skin but it could be adjusted for other tissues and applications (Wang et al., 2006b).

Referring to Fig. 2(b), the energy stored in a dualpinned lever subjected to a external force $f^{z}$ at the tip 
Submitted version. Final version to appear in the IJRR (2009).

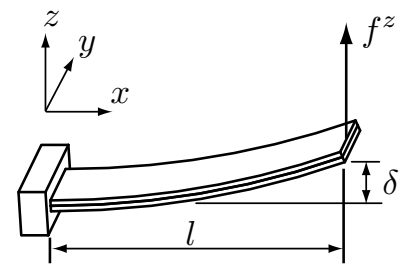

(a)

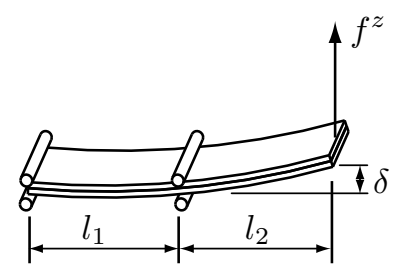

(b)
Figure 2: Two mounting methods: (a) cantilever, (b) dual-pinned lever.

is

$$
\begin{aligned}
U & =\int_{0}^{l_{1}+l_{2}} \int_{0}^{w} \int_{-h / 2}^{h / 2} u^{U} \mathrm{~d} x \mathrm{~d} y \mathrm{~d} z \\
& +\int_{0}^{l_{1}+l_{2}} \int_{0}^{w} \int_{-h / 2}^{h / 2} u^{L} \mathrm{~d} x \mathrm{~d} y \mathrm{~d} z,
\end{aligned}
$$

where $u^{U}$ and $u^{L}$ are the energy densities of the upper layer and lower layer of the beam respectively. These are written (Smits et al. 1991),

$$
\begin{aligned}
u^{U} & =\frac{1}{2 E}\left(\sigma^{U}\right)^{2}-d_{31} E_{3} \sigma^{U}+\frac{1}{2} \epsilon_{33}^{T} E_{3}^{2}, \\
u^{L} & =\frac{1}{2 E}\left(\sigma^{L}\right)^{2}+d_{31} E_{3} \sigma^{L}+\frac{1}{2} \epsilon_{33}^{T} E_{3}^{2},
\end{aligned}
$$

where $\sigma^{U}$ and $\sigma^{L}$ are the stress of the upper and lower beam layers respectively; the $\epsilon_{33}^{T}$ is the permittivity at constant stress. The bending moment caused by an external force $f^{z}$ at the tip is

$$
M^{E}(x)= \begin{cases}l_{2} / l_{1} x f^{z} & \text { if } 0 \leq x \leq l_{1}, \\ \left(l_{1}+l_{2}-x\right) f^{z} & \text { if } l_{1}<x \leq l_{1}+l_{2} .\end{cases}
$$

Therefore, the stresses in the upper and lower beam layers are respectively

$$
\begin{aligned}
& \sigma^{U}(x, z)=d_{31} E_{3} E\left(\frac{1}{4}-\frac{z}{2 h}\right)-\frac{M^{E}(x)}{I}\left(z+\frac{h}{2}\right), \\
& \sigma^{L}(x, z)=d_{31} E_{3} E\left(\frac{1}{4}-\frac{z}{2 h}\right)-\frac{M^{E}(x)}{I}\left(z-\frac{h}{2}\right),
\end{aligned}
$$

where $I$ is the moment of inertia of the beam. From Eq. (3), the total energy stored in the beam is

$$
\begin{aligned}
U= & w h E_{3}^{2}\left(l_{1}+l_{2}\right)\left(\epsilon_{33}^{2}-\frac{1}{4} d_{31}^{2} E\right) \\
& +\frac{l_{2}^{2}\left(l_{1}+l_{2}\right)}{4 E w h^{3}} f^{z 2}+\frac{3 d_{31} E_{3}}{4 h}\left(l_{1}+l_{2}\right) l_{2} f^{z} .
\end{aligned}
$$

From the energy method, the tip deflection is then

$$
\delta=\frac{\partial U}{\partial F^{z}}=\frac{\left(l_{1}+l_{2}\right) l_{2}{ }^{2}}{2 E w h^{3}} f^{z}+\frac{3 d_{31}\left(l_{1}+l_{2}\right) l_{2}}{4 h} E_{3} .
$$

In summary, the compliance and the free deflection of a dual pinned bender are

$$
\frac{\partial \delta}{\partial F^{z}}=\frac{\left(l_{1}+l_{2}\right) l_{2}^{2}}{2 E w h^{3}} \text { and } \delta_{\text {Free }}=\frac{3 d_{31}\left(l_{1}+l_{2}\right) l_{2}}{4 h^{2}} V_{\max }
$$

If we desire a cantilever bender to achieve the same free deflection as a dual-pinned bender, its length $l$ must be $\sqrt{\left(l_{1}+l_{2}\right) l_{2}}$. Its compliance, $\left[\left(l_{1}+l_{2}\right) l_{2}\right]^{3 / 2} /\left(2 E w h^{3}\right)$, is always larger than that of the dual-pinned beam which is $\left[\left(l_{1}+l_{2}\right) l_{2}^{2}\right] /\left(2 E w h^{3}\right)$. Alternatively, it is easy to show that if we want a cantilever to have same stiffness as a dual-pinned lever, its free deflection is always smaller.

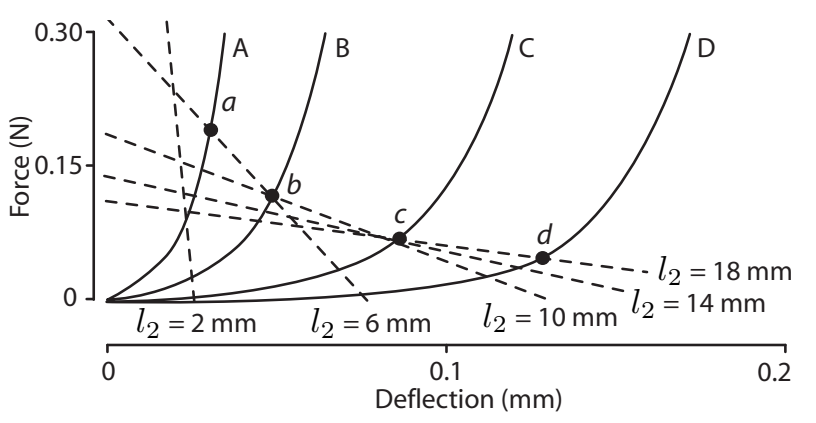

Figure 3: Illustration of optimal $l_{2}$ of a dual-pinned bender when coupling with different skins. The skin curves were derived from the data in (Wang \& Hayward, 2007).

Figure 3 shows the effect of loading a bender with a patch of skin exhibiting nonlinear stiffness with the parameters listed in Table 1. Dashed lines indicate the force-deflection relationships of benders when $l_{2}$ equals 2, 6, 10, 14, and $18 \mathrm{~mm}$ as per Eq. (10). Solid lines, A, B, C, and D, represent typical force-deflection curves exhibited by human fingerpad skin when loaded tangentially (Wang and Hayward, 2007). The intersections, which represent static equilibria, coincide with the maximum strains that can be achieved for each skin characteristic when mechanically coupled with benders of different $l_{2}$ values. Since skin has different mechanical properties for different people, the $l_{2}$ lengths that can achieve maximal strain are different. For instance, a skin with force-deflection curve $A$ reaches a maximal strain when $l_{2}$ is $6 \mathrm{~mm}$ (all the other intersections are on the left). With a skin exhibiting a force-deflection 
curve D, $l_{2}$ should be $18 \mathrm{~mm}$. It can be seen that by adjusting the value of $l_{2}$, a maximum strain can always be achieved. Specifically, if typical skins have curves like B or C (neither very soft nor very hard), then the preferred $l_{2}$ lengths ought to be from 6 to $10 \mathrm{~mm}$.

Table 1: Parameters used in Fig. 3.

\begin{tabular}{ll}
\hline$l_{1}+l_{2}$ & $30 \mathrm{~mm}$ \\
$E$ & $8 \times 10^{10} \mathrm{~N} / \mathrm{m}^{2}$ \\
$w$ & $1.6 \mathrm{~mm}$ \\
$h$ & $0.25 \mathrm{~mm}$ \\
$d_{31}$ & $1.9 \times 10^{-10} \mathrm{~m} / \mathrm{V}$ \\
$V_{\max }$ & $180 \mathrm{~V}$ \\
\hline
\end{tabular}

\subsection{Psychophysics experiment}

The approximations and the assumptions needed to arrive at a preferred bending motor clamping geometry were numerous. Therefore, a psychophysics experiment was conducted in order to validate the results. Its principle was to ask partipants to adjust the voltage activation so that they would just detect a tactile sensation for five different bender mounting geometries. This way we could determine the geometry that gave the greatest amount of sensation for the least voltage.

\subsubsection{Setup}

Referring to Fig. 4, two $1.6 \mathrm{~mm}$ wide, $30 \mathrm{~mm}$ long piezoelectric bimorph benders (Model: T215-H4-303Y, Piezo Systems Inc., Boston, MA) were dual-pinned mounted. A position sensor (Model: 602, Duncan Electronics Inc., Irvine, CA) was connected to an adjustable upper hinge that was supported by a micrometric positioning stage. The position of the upper hinge read by a personal computer after analog-to-digital conversion. Two digitalto-analog channels drove two high voltage amplifiers (Model: 3584JM; Texas Instruments Inc., Dallas, Tx, USA) to activate the benders. An aluminum gutter, whose height was also adjustable, was placed above the piezo tweezers to immobilize the subjects' fingers.

\subsubsection{Participants}

Six healthy, right-handed McGill students (2 females and 4 males) volunteered to participate in the experiment. Before the experiments, the informed consent of the participants was obtained in accordance with the

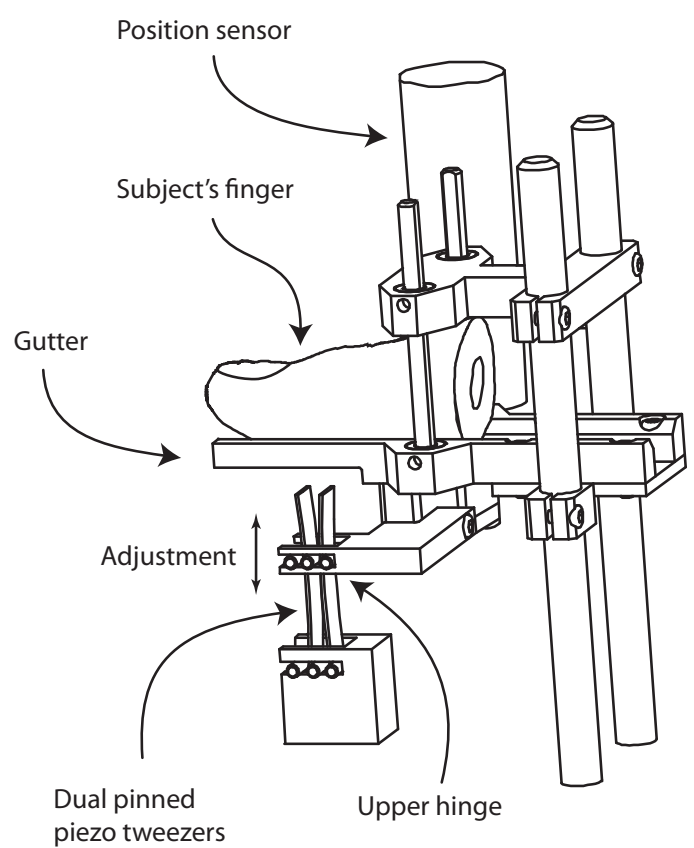

Figure 4: Stimulus generation setup.

requirements of the McGill University Policy on the Ethical Conduct of Research Involving Human Subjects.

\subsubsection{Stimulus and Protocol}

To better approximate the conditions under which a tactile transducer would actually be used, a non-periodic signal was selected as the stimulus. It consisted of a sequence of pulses shaped like the differential of a Gaussian with a duration of 0.5 seconds per pulse separated by time intervals randomly varying from 0.2 to $1.5 \mathrm{sec}-$ onds (see Fig. 5). It was hoped that this pulsed stimulus minimized sensory adaptation. It also made it impossible for participants to anticipate the next pulse, avoiding possible biases.

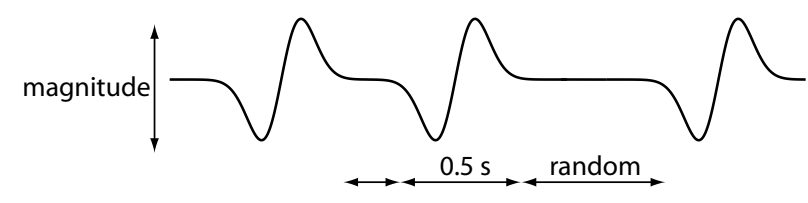

Figure 5: Stimulus.

At the start of the trials, participants were seated 
comfortably in front of the apparatus, and had their right index digit gently resting on the gutter located above the benders. The height of the gutter was then adjusted to ensure that the piezoelectic tweezers indented the fingerpad by $1 \mathrm{~mm}$. During the trials, the computer was continuously producing stimulus signals. The magnitude of the pulses was controlled by the participants by pressing the up-arrow key if they wanted to increase magnitude, or the down-arrow key if they wanted to decrease it. The upper hinge was positioned in order to set $l_{2}$ to $2,6,10,14$ and $18 \mathrm{~mm}$ in sequence. At each configuration, the participants were asked to find the minimum magnitude of the pulses that they could feel clearly. This was done by exploring a few reversals around the detection threshold.

\subsubsection{Results}

Figure 6 illustrates the mean minimum magnitudes that made the subjects distinctly feel the stimulation for the five geometries. The data show that when $l_{2}$ was 6 millimeters, the minimum intensity needed to evoke a distinct tactile sensation was the smallest. We thus expect that the deflection and hence the strain reaches its peak at this particular configuration.

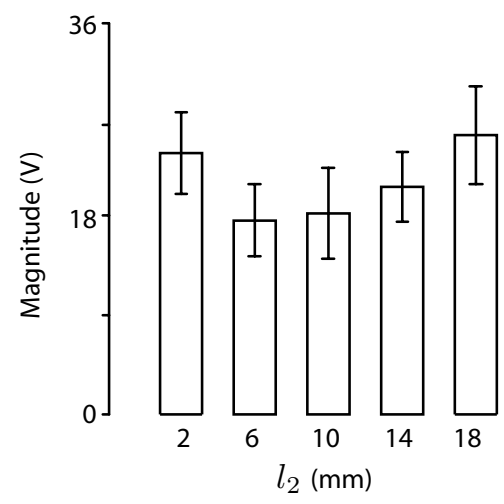

Figure 6: Results of optimal length adjustment.

One-way analysis of variance (ANOVA) showed that there were significant differences between all adjacent $l_{2}$ values $(p<0.05)$ except between $l_{2}$ equals $6 \mathrm{~mm}$ and $10 \mathrm{~mm}(p=0.783)$. This can be attributed to the fact that the maximum strain is achieved when $l_{2}=6 \mathrm{~mm}$ and $l_{2}=10 \mathrm{~mm}$, as shown in Fig. 3, point $b$.

\subsubsection{Discussion}

The biomechanics experiments reported in reference (Wang and Hayward, 2007) conducted with similar piezoelectric tweezers, but whose width was slightly wider than those used in present experiments, deformed the human fingerpad skin to larger strain when $l_{2}$ was equal to $11 \mathrm{~mm}$. In these tests, the contactors were bonded to skin. In the present psychophysical tests, stimulation was achieved by lateral traction, yet the results generally agree. Collectively, these results support the conclusion that strain is the mechanical variable that determines tactile sensations.

\section{Design and Fabrication}

The general design goals of the tactile display did not differ from what is often desired for such displays, save for the fact that the principle of operation is to apply tangential traction to the skin, rather than normal indentation. Specifically these goals were:

Modular design. Researchers who have attempted the design and construction of high density displays have often commented on the necessity to leave room for experimentation and debugging. Therefore a key requirement for an experimental design is modularity as well as assembly/disassembly convenience.

Compact and light-weight. It is also often commented that prospects for applications of tactile displays come from their ability to be integrated with forcefeedback haptic devices, with conventional data input devices, or by being directly worn with the aid, for example, of a data glove. Bulk and weight are key factors. This also holds true for the driving electronics, lest the user be forced to cope with cumbersome cable bundles.

Large deformation. Specifications for pin-type displays are often described in terms of pin displacement and exerted force. The intricate details of contact mechanics created by millimetric-scale skin indentors preclude simple descriptions in terms of their intrinsic effects on the skin. In contrast, for tangential traction displays, these specifications are more straightforward since the objective is create deformation in small patches defined by the geometry of the contactors. Direct in vivo measurements (Levesque and Hayward, 2003), informal observations (Levesque et al., 2005), as well as recent 
biomechanical data (Wang and Hayward, 2007) lead us to believe that tangential skin deformation should be in the range of $30 \%$ to $50 \%$ of strain.

Wide bandwidth. It is accepted that a general purpose tactile display system requires wide bandwidth since the mechanoreceptors embedded in human fingerpad skin are known to respond to wide range of frequencies varying from a few $\mathrm{Hz}$ to a few hundred $\mathrm{Hz}$ according to the stimulation conditions (Johnson et al. 2000).

High spatial resolution. While the nature of an effective measure of tactile acuity is a debated topic (Craig and Johson, 2000), it is well accepted that the two point discrimination threshold reflects the capabilities of perceiving tactile stimuli such as texture and Braille (Johnson and Hsiao, 1992; Boven et al., 2000). For the human fingerpad, the static two-point discrimination threshold under different mechanical stimulation conditions varies from $0.84 \mathrm{~mm}$ to $5 \mathrm{~mm}$ (Johnson and Phillips, 1981; Lamb, 1983). Consequently, the target spatial period of the display should be lower than $2 \mathrm{~mm}$.

Tactile transducer. The present configuration of the tactile transducer comprises 60 miniature piezo bimorph blades forming a $6 \times 10$ array. These dimensions were determined from experimentation, material availability and by targeting the performance figures outlined earlier. The cross section size of each blade was $0.5 \times 1.6$ millimeter, and the spatial resolution of the array was $1.2 \times 1.8$ millimeter. To construct the array, piezoelectric bimorph plates were cut to a comb shape with 6 teeth as shown in Fig. $7 .^{1}$ The geometry was similar to that used in (Pasquero and Hayward, 2003), but the dimensions were significantly revised to compensate the change of free deflection due to the dual-pinned mounting method.

The surface electrode was carefully removed in the common area at the root of the comb teeth, making them electrically independent. This resulted in "comb modules" which are illustrated in Fig. 7, where electrical connections are provided by small independent printed circuits boards (PCB). Each piezoelectric comb was bonded to these printed circuit boards with high performance epoxy and the individual connections established by soldering the electrodes using thin wires. This unit formed

\footnotetext{
${ }^{1}$ From the time of initial submission of this article and the present revision, several other models have been manufactured with a $8 \times 8$ configuration, that is, 8 combs with 8 teeth.
}

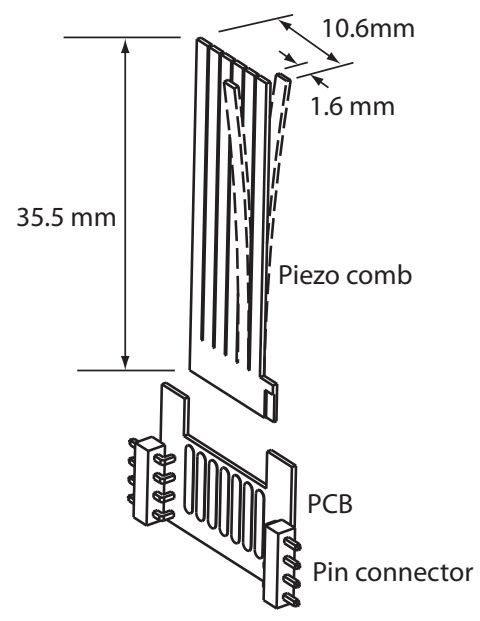

Figure 7: Single piezo comb module.

a complete interchangeable module with six (or eight) actuators.

Looking at Fig. 8, modules were assembled in a "fan" geometry which had the effect of creating a cradle of radius $25 \mathrm{~mm}$ upon which the fingertip could rest with minimal pre-loading. To protect users from electrical shock, a thin layer of varnish was coated at the tip of each piezo tooth. Moreover, the surface electrode within $0.5 \mathrm{~mm}$ from the each tooth tip was removed. It is should be noted that four-pin edge connectors, as shown in Fig. 7, should not be considered to be rigid clamps. The metallic square pins insert into miniature spring-loaded clamps that allow some freedom, enabling the connection to be approximated by a hinge for small angular movements.

Compactness and modularity. The actuator array is the most fragile and expensive part of the display. The entire display is illustrated in Figs. 8 and 9. Each module is connected to two side-boards via two fourpin connectors. These side-boards have two functions. The first is to serve as structural mechanical support in the form of a stiff box-structure needed to provide support to the dual-pinned benders. The second is to route signals from the driving electronics located on the base motherboard. The boards also contain electrical test pads for debugging. In addition, pre-drilled holes at certain places make it possible to modify the ratio $l_{2} / l_{1}$. In the current design, the holes were pre-drilled to allow for $l_{2}=7.5 \mathrm{~mm}$ and $l_{2}=10.0 \mathrm{~mm}$. But other hole patterns could easily accommodate other $l_{2}$ values. 
Submitted version. Final version to appear in the IJRR (2009).

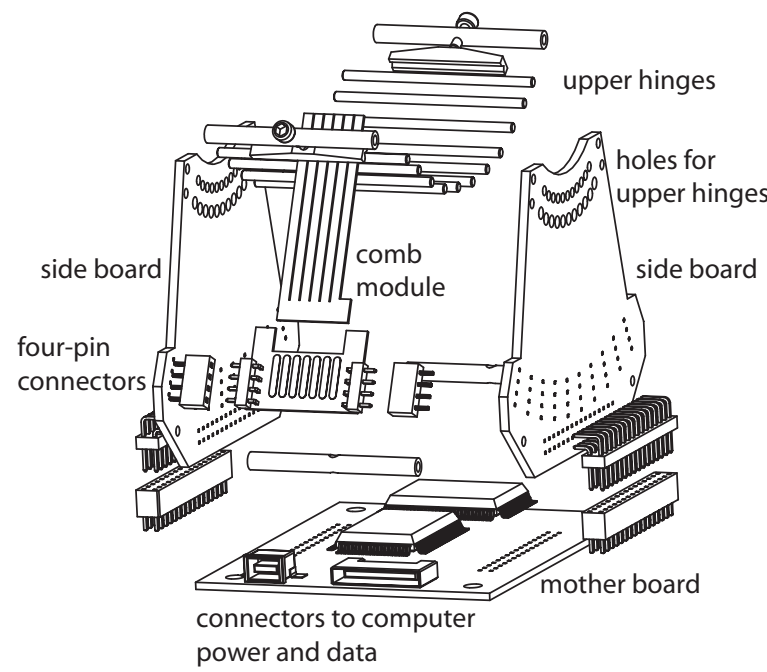

Figure 8: Exploded view of the tactile display.

An assembled view of the whole system is seen in Fig. 9 along with the connectors to a computer interface to the power management unit.

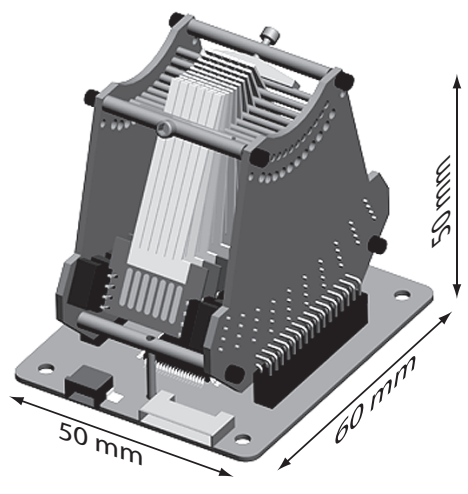

Figure 9: Assembled view of the tactile display.

Driving electronics. In order to maximize portability and reduce the number of connections, we applied the principle of time division to the greatest extent possible. For this, we used two HV257 chips (Supertex Inc., Sunnyvale, CA, USA). Each chip drives 30 (or 32) actuators. This chip is an integrated 32-channel sample/hold and high voltage amplifier. The system diagram is illustrated in some detail in Fig. 10 where, for the sake of conciseness, only one HV257 is shown. The computer first sends a desired analog output through a DAC, then specifies a channel address of the high-voltage amplifier. Since the computer refreshes each channel at a frequency of $4 \mathrm{kHz}$, which is much higher than the cut-off frequency of the sample-and-hold circuits, the output signal from each channel is well represented. Moreover, at this frequency, the electromechanical transfer function of the actuators acts as a reasonably good reconstruction filter. Nevertheless, a noticeable improvement in the transparency of the device was obtained by inserting first-order low-pass filters (200 $\mathrm{Hz}$ cut-off) between the amplifiers and the actuators in an effort to improve signal reconstruction. These are indicated in Fig. 10. Physically, they were realized by surface-mount components supported by the miniature printed circuit boards shown in Fig. 7.

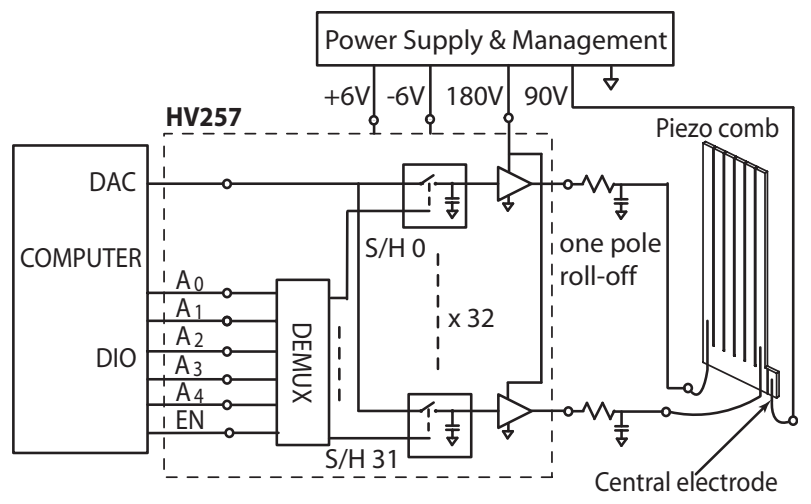

Figure 10: Driving electronics diagram.

Since the HV257 operates unipolar amplifiers, we connected the central electrode of all piezo combs to a regulated $90 \mathrm{~V}$ center-tap power rail and the amplifiers supplied voltages between 0 and $180 \mathrm{~V}$. The potential difference between the surface electrode and central electrode thus varied from -90 to $+90 \mathrm{~V}$. Current limiters internal to the chip made the system completely safe to use.

Software. Here, we used RTLinux ${ }^{\mathrm{TM}}$ to drive the system from hard realtime clocks. Two realtime tasks, the tactile rendering task and the refreshing task, were runing simultaneously to interact with human subjects and drive the device. The tactile rendering task, which is in charge of the interaction between human and virtual environments, generated tactile signals at $4 \mathrm{kHz}$. These signals were then sent to the refreshing task through a FIFO. This task, which ran at $200 \mathrm{kHz}$, finished refreshing all channels in 30 consecutive clock cycles. 


\section{Performance Assessment}

To measure the performance of the actuators of the display in terms of free deflection, stiffness, blocked force, and bandwidth, five teeth were randomly selected and tested. The measurements were conducted under two test conditions, $l_{2}=7.5 \mathrm{~mm}$ and $l_{2}=10.0 \mathrm{~mm}$.

\subsection{Free deflection}

To measure the deflection of a tooth, a piezo comb was vertically dual-pinned mounted in the apparatus shown in Fig. 4, and a tiny mirror was glued to the tip of a tooth in order to reflect a laser beam toward a lateral position sensing detector (PSD, Model DL-10; UDT Sensors, Inc., Hawthorne, CA, USA). When the maximum voltage, i.e. $\pm 90 \mathrm{~V}$, was applied, the shift of the bright spot created by the reflected beam on the PSD surface was measured, giving tooth deflection after calibration. Ten cycles of maximum voltage were applied, and the measured deflections were averaged. It was $0.0752 \pm 0.0012 \mathrm{~mm}$ for $l_{2}=7.5 \mathrm{~mm}$, and $0.104 \pm 0.001 \mathrm{~mm}$ for $l_{2}=10.0 \mathrm{~mm}$.

\subsection{Blocked force and stiffness}

To measure the blocked force, we first applied the maximum voltage to a piezo tooth, and then gradually increased a tangential force applied at the tip of the tooth until the deflection was zeroed. This procedure was repeated for ten cycles. The blocked force produced by each tooth was $0.224 \pm 0.01 \mathrm{~N}$ for $l_{2}=7.5 \mathrm{~mm}$, and $0.147 \pm 0.01 \mathrm{~N}$ for $l_{2}=10.0 \mathrm{~mm}$.

The stiffness was assessed by measuring the relationship between deflections and applied forces. The setup was similar to that used to measure the blocked force, but no voltage was applied to the piezo tooth. Figure 11 illustrates the results.

\subsection{Bandwidth}

The free displacement frequency response of a tooth was measured by sweeping a sinusoidal input signal, which was amplified by the HV257, between $30 \mathrm{~Hz}$ to $2000 \mathrm{~Hz}$ and by measuring the resulting deflection. The results, see Fig. 12, show that system bandwidth is larger than $250 \mathrm{~Hz}$. The actuator resonates at about $1.5 \mathrm{kHz}$ when $l_{2}=10 \mathrm{~mm}$, and at $1750 \mathrm{~Hz}$ when $l_{2}=7.5 \mathrm{~mm}$. Since the bandwidth of the HV257 op. amp. channels is $4 \mathrm{KHz}$, the artifacts caused by the HV257 in the bode plot are negligible. In both cases, this frequency is far above the

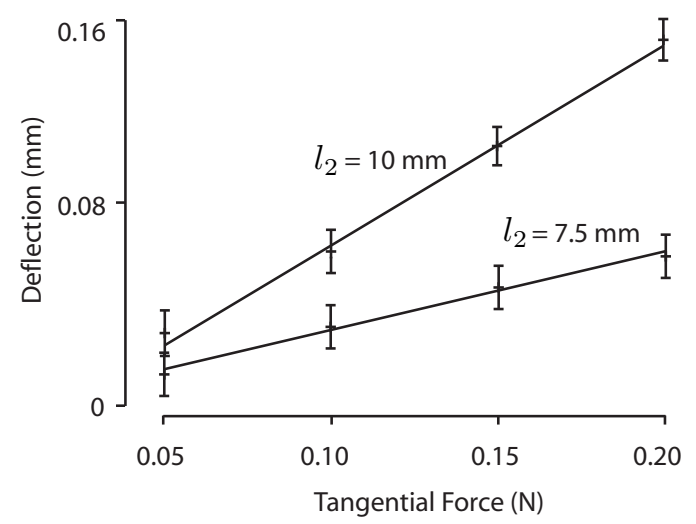

Figure 11: Measured tooth deflection for two different $l_{2} / l_{1}$ ratios.

targeted operating range. The amplitude roll-off starting at about $250 \mathrm{~Hz}$ can be attributed to a combination of internal dissipation in the piezo-electric material and losses in the hinges, which are presently made of thermoplastic sheathes heat-shrunk around stiff ceramic rods. Initial tests indicated that the high-Q resonance present at $1.5 \mathrm{kHz}$ had a significant effect on the tactile transparency of the device, especially when the driving signal contains transients. It is therefore recommended to employ roll-off filters as in Fig. 10.

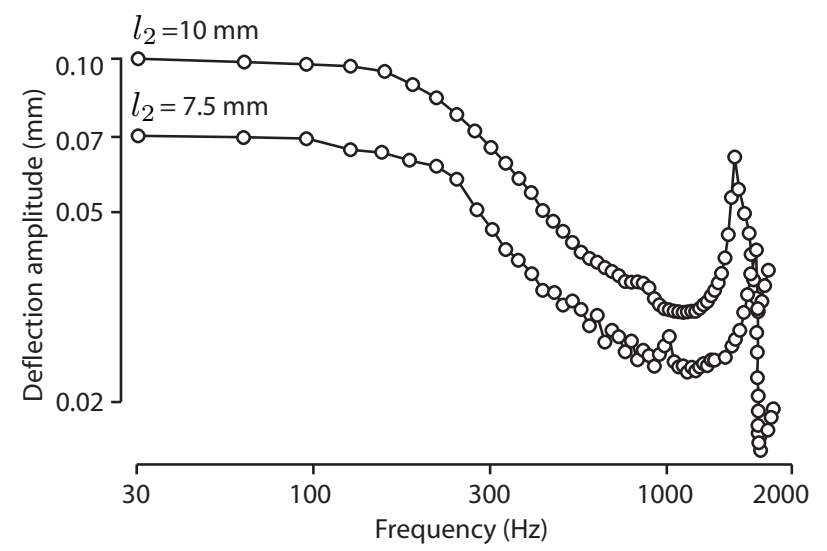

Figure 12: Measured Bode plot for different $l_{2} / l_{1}$ ratios. 


\section{Preliminary Tests}

The device was successfully demonstrated to a few hundred visitors at the 2006 ACM Conference on Human Factors in Computing Systems (CHI 2006), with a pilot application involving the recognition of tactile graphics (Wang et al. 2006a).

Experiments were nevertheless conducted to further assess the effectiveness of the device in terms of its ability to deliver meaningful tactile sensations. The device was configured with $l_{2}=7.5 \mathrm{~mm}$ and programmed to create lines of tactile dots. Participants were asked to detect simple geometrical relationships between two lines: their mutual angle and the place they met. The choice of a detection task rather than a discrimination task was justified by the desire to evaluate the capacity of the device to create easily detectable percepts, rather than to test the sensory performance of the participants.

\section{$5.1 \quad$ Participants}

Six healthy, right-handed participants, including 2 females and 4 males, were recruited among the McGill University graduate students and research staff with age of $32 \pm 5$ years. The participation was voluntary. Before the experiments, informed consent was obtained in accordance with the requirements of the McGill University Policy on the Ethical Conduct of Research Involving Human Subjects.

\subsection{Tactile rendering}

Figure 13 illustrates the experimental setup. The tactile transducer was mounted on a Pantograph haptic device (Campion et al., 2005), which was used here as a passive two-dimensional frictionless planar carrier. A personal computer with RTLinux ${ }^{\mathrm{TM}}$ operating system sampled the position of the Pantograph at $1 \mathrm{kHz}$ and the position of the tactile transducer array was derived according to the kinematics of the mechanism. The virtual environment software generated tactile signals. These were relayed to the tactile display as in Fig. 10 .

Subjects explored a $113 \times 60 \mathrm{~mm}$ virtual surface by moving the display within the carrier's workspace with their index digit. Exploration was effortlessly accomplished due to the near absence of friction in the guiding mechanism. The movements were recorded by the computer with a spatial resolution of $10 \mu \mathrm{m}$, and temporal resolution of $1 \mathrm{~ms}$. During the experiment, the fingerpad remained fixed to the top of the transducer array and could guide it without the help of any attachment. The virtual surface was divided into $94 \times 33$ cells. The size of each cell was the same as the spatial resolution of the tactile display, i.e. $1.2 \times 1.8 \mathrm{~mm}$.

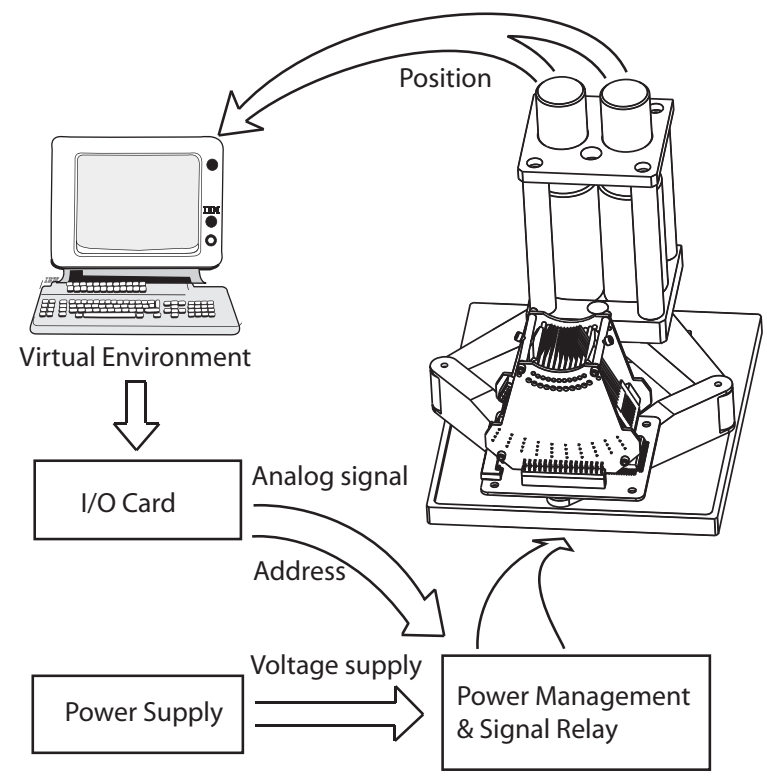

Figure 13: Experimental setup for rendering.

The lines were composed of small dots located at the center of the cells as shown in Fig. 14(a). The effectiveness of a similar method was demonstrated when creating virtual Braille dots (Levesque et al., 2005) and virtual textures (Wang et al. 2006a). Along the mediallateral $x$ axis, when a piezo actuator was in the territory of a virtual dot, the actuator deformed the fingerpad skin laterally according to its position relative to the origin of the dot, see Fig. 14(b). The method employed here differs slightly from that described in the above references (full wave instead of a half-wave), but the resulting sensations were similar. The actuator activating voltage $V_{\text {act }}$ was:

$$
V_{\text {act }}=V_{0} \sin \left(2 \pi \frac{x-x_{\text {orig }}}{d}\right),
$$

where $V_{0}$ is the amplitude, $d$ the diameter of the virtual dot, $x$ the position of the piezo actuator, and the $x_{\text {orig }}$ is the position of the virtual dot. Along the rostral-caudal $y$ axis, the piezo actuator is mapped to the closest cell in that direction. Since the size of the cells matches the 
pitch of the tactile transducer array, the actuators were consistently mapped to the closest cells.

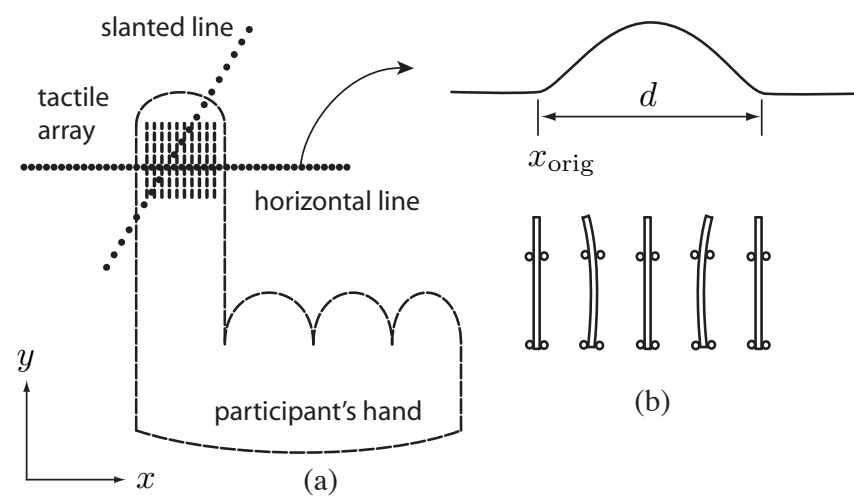

Figure 14: (a) Virtual texture composed of dots. (b) Virtual dot rendering.

\subsection{Protocol}

For each trial, two virtual lines, one horizontal and one slanted, were randomly generated by the computer. The amplitude, $V_{0}$, used for rendering the lines was was randomly set among the values $10 \mathrm{~V}, 20 \mathrm{~V}, 30 \mathrm{~V}, \cdots, 90 \mathrm{~V}$ for each trial. The $y$ coordinate of the horizontal line was randomly set between $15 \mathrm{~mm}$ and $45 \mathrm{~mm}$. The angle of the slant line was randomly chosen among the values of 30, 60, 90, 120, and 150 degrees. The intersection of two lines was also randomly set. The task was to localize the intersection of the two lines and to judge the orientation of the slanted line.

At the start of the trials, participants were seated comfortably in front of the apparatus, and had their index digit rest on the active surface of the tactile transducer array. They were informed of the five possible slanted line orientations. During the trials, subjects gently explored the virtual surface to feel the virtual lines. The velocity of the movement was always smaller than $50 \mathrm{~mm} / \mathrm{s}$. As soon as subjects thought they had localized the intersection of two lines, they were instructed to move the center of the tactile array to that position and to press the space bar of the computer keyboard. They also had to type in the judged angle of the slanted line. The subjects were asked to complete each trial within 10 seconds. Each subject performed 150 trials.

\subsection{Results and discussion}

To evaluate the human performance, for each amplitude $V_{0}$ value and each angle of the slant line, the spatial errors between the real intersection point and the localized intersection point were averaged as well as the error between the real and perceived angle of the virtual slant line. Figure 15 summarizes the results.
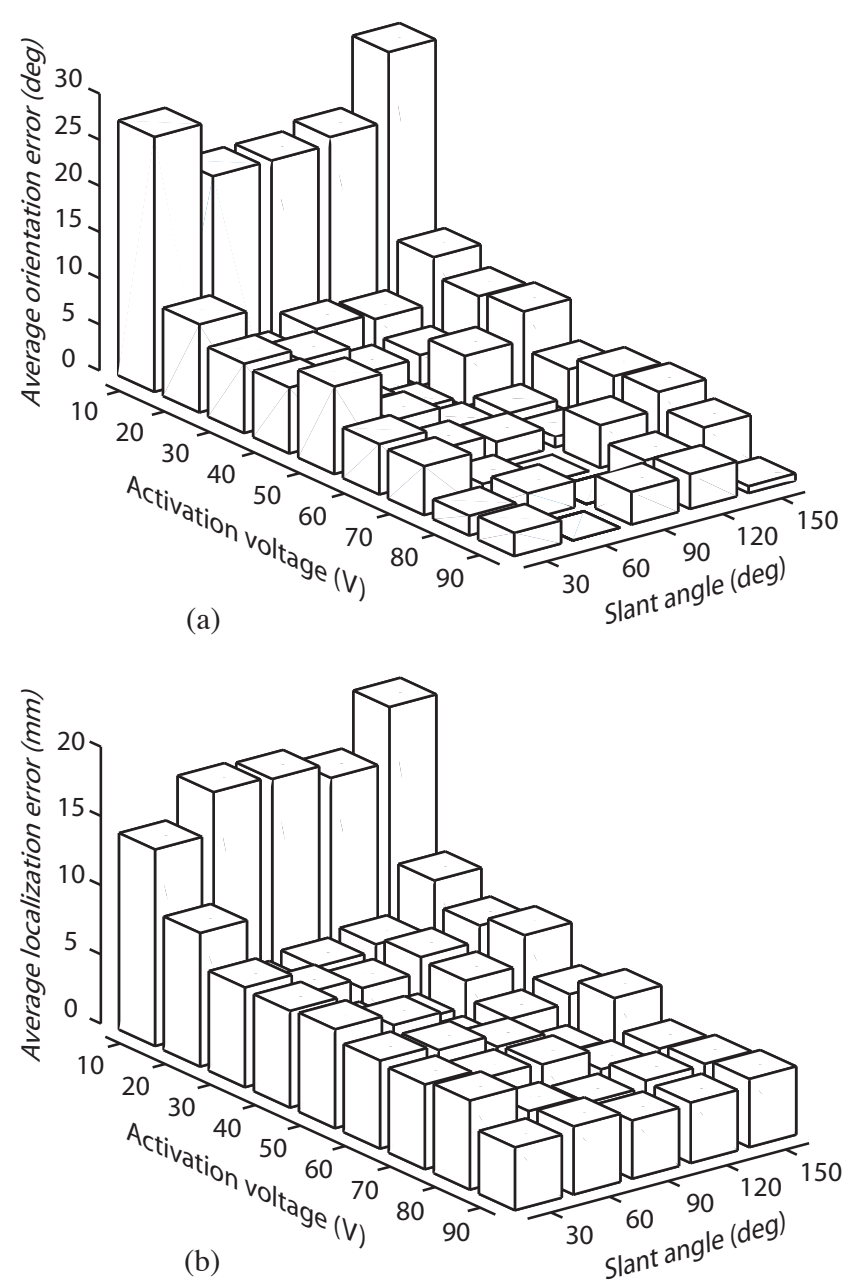

Figure 15: (a) Averaged orientation error in judging the orientation of virtual slant lines. (b) Averaged localization error in localizing the intersection of two virtual lines

When the maximum applied voltage $V_{0}$ was $10 \mathrm{~V}$, the performance was low. The averaged orientation detection error was around $25^{\circ}$, and the averaged localization error was around $15 \mathrm{~mm}$. However, when $V_{0}$ increased to $20 \mathrm{~V}$, the performance improved dramatically. The per- 
formance was then consistent when $V_{0}$ was larger than $20 \mathrm{~V}$, demonstrating that the device is capable of rendering perceivable virtual features. The minimum $V_{0}$ for good stimulation agrees well with the result of psychophysical experiments presented in (Wang and Hayward, 2006), which showed that the $V_{0}$ needed to provide meaningful tactile sensations was $15 \mathrm{~V}$. When the minimum $V_{0}$ was substantially higher, the averaged orientation error and localization error remained at $5^{\circ}$ and $5 \mathrm{~mm}$, respectively. The relatively poor performance in locating a feature under the finger may be attributed to the difficulty of the task rather than factors related to the transducer. The reader may consult (Levesque et al., 2007; Levesque and Hayward, 2008) for a more complete assessment of the expressive capabilities of the device.

\section{Conclusion and future work}

This paper described a portable, modular, highperformance tactile display device based on lateral skin deformation. It has 60 actuators creating an active surface of about $100 \mathrm{~mm}^{2}$ in the shape of a cradle designed to minimize the finger pre-load when the tactile signals are quiescent. These actuators were optimized to maximize tangential strain in average glabrous skin. The system is compact $\left(150 \mathrm{~cm}^{3}, 60 \mathrm{~g}\right)$ and has integrated electronics requiring a small number of wires for operation. A prominent feature of the device is the ease with which it can be fabricated and maintained compared to earlier generations.

The results indicate that each actuator could produce a free deflection of $0.1 \mathrm{~mm}$ and a blocked force of $0.15 \mathrm{~N}$ (or $0.075 \mathrm{~mm}$ and $0.22 \mathrm{~N}$ ), which appear to be capable of creating large deformation in $1.6 \times 1.0 \mathrm{~mm}$ patches of the fingertip skin for most users. Furthermore, the system bandwidth is high, more than $250 \mathrm{~Hz}$, making the system not only promising for the creation of the sensations of small shapes, but also of fine textures.

Psychophysical experiments validated the effectiveness of the device in rendering virtual textures. Experimental results indicated that the threshold of creation of a tactile sensation at a single site was in the vicinity of $20 \mathrm{~V}$, while the maximum activation voltage can be up to $90 \mathrm{~V}$, allowing for significant headroom in the delivery of tactile signals.

Our present efforts are directed at further characterization of the device and at the development of a variety of activation patterns designed to explore the range of sensations that can be created with this technology.
Some recent results obtained by taking advantage of the fine spatial and temporal capabilities of the device can be found in (Petit et al., 2008; Carter et al., 2008; Konkle et al., 2009).

\section{Acknowledgment}

The authors are indebted to Jérôme Pasquero and Vincent Levesque of the Haptics Laboratory at McGill University who were instrumental in motivating us to develop this new device. The authors thank Garrett Stanley and Daniel Millard for their comments on revised manuscript. This research was supported IRIS, the Institute for Robotics and Intelligent Systems. Qi Wang would like to thank McGill University for an Eric L. Adler Fellowship in Electrical Engineering and Vincent Hayward would like to thank the Natural Sciences and Engineering Research Council of Canada (NSERC) for a Discovery Grant.

\section{References}

André, T., Lefèvre, P. and Thonnard, J.-L. (2009). A continuous measure of fingertip friction during precision grip. Journal of Neuroscience Methods, 179(2): 224-229.

Biggs, J. and Srinivasan, M. A (2002). Tangential versus normal displacements of skin: Relative effectiveness for producing tactile sensations. Proceedings of the 10th International Symposium on Haptic Interfaces for Virtual Environment and Teleoperator Systems, pp. 121-128.

Bliss, J., Katcher, M., Rogers, C. and Shepard, R (1970). Optical-to-tactile image conversion for the blind. IEEE Transactions on Man-Machine Systems, 11(1): 58-65.

Van Boven, R. W., Hamilton, R. H., Kauffman, T., Keenan, J. P. and Pascual-Leone, A. (2000). Tactile spatial resolution in blind braille readers. Neurology, 54: 2230-2236.

Campion, G., Wang, Q., and Hayward, V. (2005). The Pantograph Mk-II: A haptic instrument. Proceedings of the IEEE/RSJ International Conference on Intelligent Robots and Systems, IROS'O5, pp. 723728. 
Carter, O., Konkle, T., Wang, Q., Hayward, V. and Moore, C. I. (2008). Tactile rivalry demonstrated with an ambiguous apparent-motion quartet. Current Biology, 18(14): 1050-1054.

Craig, J. C., and Johnson, K. O. (2000). The two-point threshold: Not a measure of tactile spatial resolution. Current Directions in Psychological Science, 9: $29-32$.

Drewing, K., Fritschi, M., Zopf, R., Ernst, M. O., and Buss, M. (2005). First evaluation of a novel tactile display exerting shear force via lateral displacement. ACM Transactions on Applied Perception, 2(2): 118-131, 2005.

Ghodssi, R., Beebe, D. J., White, V., and Denton, D. D. (1998). Development of a tangential tactor using a LIGA/MEMS linear microactuator technology. Proceedings of the MicroElectro-Mechanical Systems (MEMS). International Mechanical Engineering Congress and Exposition, Microelectromechanical Systems, Vol. DSC 59, pp. 379-386.

Hasser, C. J. and Weisenberger, J. M. (1993). Preliminary evaluation of a shape-memory-alloy tactile feedback display. Proceedings of the Symposium on Haptic Interfaces in Virtual Environments and Teleoperator Systems, ASME Winter Annual Meeting, Vol. DSC 49, pages 73-80, 1993.

Hayward, V. and Cruz-Hernandez, M. (2000). Tactile display device using distributed lateral skin stretch. Proceedings of the Haptic Interfaces for Virtual Environment and Teleoperator Systems Symposium, Vol. DSC 69-2, pp. 1309-1314, 2000.

Iwamoto, T. and Shinoda, H (2005). Ultrasound tactile display for stress field reproduction - examination of non-vibratory tactile apparent movement. Proceedings of the First Joint Eurohaptics Conference and Symposium on Haptic Interfaces for Virtual Environment and Teleoperator Systems, World Haptics 2005, pp. 220-228.

Johnson, K. O. and Hsiao, S. S. (1992). Neural mechanisms of tactual form and texture perception. $A n$ nual Review of Neuroscience, 15: 227-250.

Johnson, K. O. and Phillips, J. R. (1981). Tactile spatial resolution: I. Two-point discrimination, gap detection, grating resolution, and letter recognition. Journal of Neurophysiology, 46: 1177-1192.
Johnson, K. O., Yoshioka, T. and Vega-Bermudez, F. (2000). Tactile functions of mechanoreceptive afferents innervating the hand. Journal of Clinical Neurophysiology, 17: 539-558.

Jungmann, M. and Schlaak, H. F. (2002). Miniaturized electrostatic tactile display with high structural compliance. Proceedings of Eurohaptics 2002, pp. $12-17$.

Benali-Khoudja, M., Hafez, M., Alexandre, J.-M., Kheddar, A. and Moreau, V. (2004). Vital: A new low-cost vibro-tactile display system. Proceedings of the IEEE International Conference on Robotics and Automation, Vol. 1, pp. 721-726.

Kikuuwe, R., Sano, A., Mochiyama, H., Takesue, N. and Fujimoto, H. (2005). Enhancing haptic detection of surface undulation. ACM Transactions on Applied Perception, 2(1): 46-67, 2005.

Konkle, T., Wang, Q., Hayward, V. and Moore, C. I. (2009). Motion aftereffects transfer between touch and vision. Current Biology, 19(9): 745-750.

Konyo, M., Tadokoro, S. and Takamori, T. (2000). Artificial tactile feel display using soft gel actuators. Proceedings of IEEE International Conference on Robotics and Automation, pp. 3416-3421.

Lamb, G. D. (1983). Tactile discrimination of textured surfaces: Psychophysical performance measurements in humans. Journal of Physiology, 338: 551565 .

Levesque, V. and Hayward, V. (2003). Experimental evidence of lateral skin strain during tactile exploration. Proceedings of Eurohaptics 2003, pp. 261275.

Levesque, V. and Hayward. V. (2008). Tactile graphics rendering using three laterotactile drawing primitives. Proceedings of the 16th Symposium on Haptic Interfaces For Virtual Environment And Teleoperator Systems, pp. 429-436.

Levesque, V., Pasquero, J. and Hayward, V. (2007). Braille display by lateral skin deformation with the stress ${ }^{2}$ tactile transducer. Proceedings of the Second Joint Eurohaptics Conference and Symposium on Haptic Interfaces for Virtual Environment and Teleoperator Systems, World Haptics 200\%, pp. $115-120$. 
Levesque, V., Pasquero, J., Hayward, V. and Legault, M. (2005). Display of virtual Braille dots by lateral skin deformation: Feasibility study. ACM Transactions on Applied Perception, 2(2): 132-149.

Moy, G., Wagner, C. and Fearing, R. S. (2000) A compliant tactile display for teletaction. Proceedings of the IEEE International Conference on Robotics and Automation, pp. 3409-3415.

Pasquero, J. (2006). Survey on communication through touch. Technical Report TR-CIM 06.04, Center for Intelligent Machines - McGill University.

Pasquero, J. and Hayward, V. (2003). STRESS: A practical tactile display system with one millimeter spatial resolution and $700 \mathrm{~Hz}$ refresh rate. Proceedings of Eurohaptics, pages 94-110.

Pasquero, J., Luk, J., Levesque, V., Wang, Q., Hayward, V. and MacLean, K. E. (2007). Haptically enabled handheld information display with distributed tactile transducer. IEEE Transactions on Multimedia, 9(4): 746-753.

Petit, G., Dufresne, A., Levesque, V., Hayward, V. and Trudeau, N. (2008). Refreshable tactile graphics applied to schoolbook illustrations for students with visual impairment. Proceedings of the 10th International ACM SIGACCESS Conference on Computers and Accessibility, ASSETS 2008, pp. 89-96.

Sarakoglou, I., Tsagarakis, N. and Caldwell, D. G. (2005). A portable fingertip tactile feedback array: Transmission system reliability and modelling. Proceedings of the First Joint Eurohaptics Conference and Symposium on Haptic Interfaces for Virtual Environment and Teleoperator Systems, World Haptics 2005, pp. 547-548.

Smits, J. G., Dalke, S. I. and Cooney, T. K. (1991). The constituent equations of piezoelectric bimorphs. Sensors and Actuators A, 28: 41-61.

Tang, H. and Beebe, D. J. (1998). A microfabricated electrostatic haptic display for persons with visual impairments. IEEE Transactions on Rehabilitation Engineering, 6(3): 241-248.

Velazquez, R., Pissaloux, E., Hafez, M. and Szewczyk, J. (2005). A low-cost highly-portable tactile display based on shape memory alloy micro-actuators. Proceedings of the IEEE International Conference on Virtual Environments, Human-Computer Interfaces, and Measurement Systems, VECIMS 2005, pp. $121-126$.

Wagner, C. R., Lederman, S. J. and Howe, R. D. (2004). Design and performance of a tactile shape display using RC servomotors. Haptics-e, 3(4).

Wang, Q. and Hayward, V. (2006). Compact, portable, modular, high-performance, distributed tactile transducer device based on lateral skin deformation. Proceedings of the Symposium on Haptic Interfaces For Virtual Environment And Teleoperator Systems, pp. 67-72.

Wang, Q. and Hayward, V. (2007). In vivo biomechanics of the fingerpad skin under local tangential traction. Journal of Biomechanics, 40(4): 851-860,

Wang, Q. and Hayward, V. (2008). Tactile synthesis and perceptual inverse problems seen from the view point of contact mechanics. ACM Transactions on Applied Perception, 5(2): 1-19.

Wang, Q., Kong, L., Sprigle, S. and Hayward, V. (2006b). Portable gage for pressure ulcer detection. Proceedings of the IEEE Engineering in Medicine and Biology Society Conference, EMBC'06, pp. 5997-6000.

Wang, Q., Levesque, V., Pasquero, J. and Hayward, V. (2006a). A haptic memory game using the $\mathrm{STReSS}^{2}$ tactile display. Proceedings of the ACM 2006 Conference on Human Factors in Computing Systems, CHI 2006, pp. 271-274.

Wellman, P., Peine, W., Favalora, G. and Howe, R. D. (1998). Mechanical design and control of a highbandwidth shape memory alloy tactile display. Experimental Robotics $V$, Casals, A., de Almeida, A. T. (eds.) (Lecture Notes in Control and Information Science, Vol. 232), pp. 56-66. Springer Verlag. 